\title{
COMPARISON OF CLOSED-FORM SOLUTIONS TO EXPERIMENTAL MAGNETIC FORCE BETWEEN TWO CYLINDRICAL MAGNETS
}

\author{
Sampart Cheedket \\ Department of Physics, School of Science \\ Center of Excellence in Plasma Science and Electromagnetic Waves (PEwave) ${ }^{1}$ \\ Chitnarong Sirisathitkul $\bowtie$ \\ Department of Physics, School of Science \\ Center of Excellence in Functional Materials and Nanotechnology ${ }^{1}$ \\ chitnarong.siri@gmail.com \\ ${ }^{1}$ Walailak University \\ 222 Thaiburi Sub-District, Thasala District, Nakhon Si Thammarat, Thailand, 80160
}

$\triangle$ Corresponding author

\begin{abstract}
The force between permanent magnets implemented in many engineering devices remains an intriguing problem in basic physics. The variation of magnetic force with the distance $x$ between a pair of magnets cannot usually be approximated as $x^{-4}$ because of the dipole nature and geometry of magnets. In this work, the force between two identical cylindrical magnets is accurately described by a closed-form solution. The analytical model assumes that the magnets are uniformly magnetized along their length. The calculation, based on the magnetic field exerted by one magnet on the other along the direction of their orientation, shows a reduction in the magnetic force with the distance $x$ and a dependence on the size parameters of magnets. To verify the equation, the experiment was set up by placing two cylindrical neodymium iron boron type magnets in a vertical tube. The repulsive force between the identical upper and lower magnets of $2.5 \mathrm{~cm}$ in diameter and $7.5 \mathrm{~cm}$ in length was measured from the weight on the top of the upper magnet. The resulting separation between the magnets was recorded as $x$. The forces measured at $x=0.004-0.037 \mathrm{~m}$ differ from the values calculated using the analytic solution by $-0.55 \%$ to $-13.60 \%$. The calculation also gives rise to a practical remnant magnetic field of $1.206 \mathrm{~T}$. When $x$ is much larger than the length of magnets, the equation of force is approximated as a simple form proportional to $1 / x^{-4}$. The finding can be directly used in magnetic levitation as well as applied in calculating magnetic fields and forces in other systems incorporating permanent magnets.
\end{abstract}

Keywords: magnetic force, permanent magnets, closed-form solution, elliptic function, nonlinear least square fitting.

DOI: $10.21303 / 2461-4262.2021 .001955$

\section{Introduction}

Magnetic fields from permanent magnets are essential for the operation of electrical generators and motors. The electromotive force in generators and the angular velocity in motors are increased with changing magnetic fluxes. Furthermore, the optimized implementation of magnets is key to novel wind turbines, flywheels, electric cars, and magnetic refrigerators which are energyefficient and eco-friendly. Since the early experiments on permanent magnets [1], the magnetic force has been a topic of fundamental interest. Despite its currently ubiquitous and advanced applications, equations describing the force exerted from cuboidal and cylindrical magnets remain under active investigations [2-8]. The calculations have also been extended to magnets in arrays and devices $[9,10]$. In addition to analytic solutions, magnetic field distributions have increasingly been simulated by the finite element method for permanent magnets of a variety of geometries such as cuboid [11] ring [12] and disk [13].

Unlike electric forces between electric charges, the magnetic forces between bars of magnets cannot be written in a simple expression because they are forces between dipoles. If the magnets are approximated as the point-like magnetic dipole moments of $m_{1}$ and $m_{2}$, the force $\left(F_{1}\right)$ between the magnets with a large separation distance $(x)$ is given as:

$$
F_{1}=\frac{3 \mu_{0} m_{1} m_{2}}{2 \pi x^{4}}
$$


where $\mu_{0}=4 \pi \cdot 10^{-7} \mathrm{~T} \cdot \mathrm{m} / \mathrm{A}$. However, the magnets in real experiments and devices are quite far from point-like objects so that the attempts to fit the experimental result with equation (1) are usually failed. The force is increasingly sensitive to geometries of the magnets when $x$ is small in comparison to the magnet size.

In this work, let's focus on magnetic forces between two cylindrical magnet bars. A closedform of magnetic forces is investigated as a function of the distance $x$ and compared to experimental results. In addition to fundamental physics, the presented equation can be directly implemented in the magnetic levitation. The calculation which is more accurate than the approximation in (1) is also useful in designing other engineering devices and systems.

\section{Materials and methods}

The experiment was set up by placing two magnetic cylinders in a vertical tube. These identical rare-earth magnets are neodymium iron boron type of $2.5 \mathrm{~cm}$ in diameter and $7.5 \mathrm{~cm}$ in length. The arrangement of magnets, magnetization directions, and the separation distance between magnets is schematically shown in Fig. 1. The bottom of the lower magnet is located on $z=0$. The magnetization of the lower magnet is $M_{1}$ with a direction pointing upward while the magnetization of the upper magnet is $M_{2}$ with a downward direction. The $M_{1}$ and $M_{2}$ are equal in magnitude. The separation distance between two magnets is measured from the top pole-face of the lower magnet and the bottom pole-face of the upper magnet. The force exerted between the upper and lower magnets was varied by changing the weight on the top of the upper magnet and the resulting separation between the magnets was recorded as $x$. The smallest $x$ of $0.004 \mathrm{~m}$ was obtained by adding the weight of the water container to $20 \mathrm{~kg}$ and the largest $x$ was $0.037 \mathrm{~m}$. The repulsive force was then plotted against the distance $x$. The nonlinear least square fitting method in [14] was applied on Mathematica to compare the forces from the equations to the experimental results. The percentage difference was determined from the difference between two values divided by the average of the two values and then converted into percentage. The forces when $x>0.037 \mathrm{~m}$ and $x<0.004 \mathrm{~m}$ including the contact force $(x=0 \mathrm{~m})$ can be predicted.

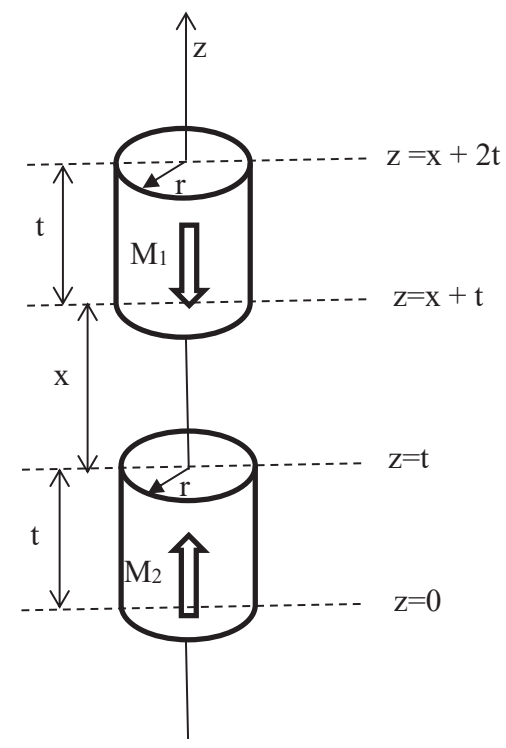

Fig. 1. Schematic diagram of two cylindrical magnets (the radius, $r=0.0125 \mathrm{~m}$ and the length, $t=0.075 \mathrm{~m}$ ) in a vertical alignment. The $z$-axis passes through the center line of both magnets

\section{Results}

The magnetic force measured from the experiment set-up exhibits the decrease with increasing $x$ from 0.004 to $0.037 \mathrm{~m}$. In Fig. 2, the experimental result can be fitted with elliptic functions of repulsive force $F_{2}$ in (2). By defining the variables as: 


$$
\begin{aligned}
& \tau=\frac{t}{2 r}, z=\frac{x+t}{r}, k=\sqrt{\frac{4}{4+\omega^{2}}}, l_{1}=\frac{1}{\sqrt{1+4 \tau^{2}}}, M_{1}=-M_{2}=M=\frac{B_{0}}{\mu_{0}}, \\
& A_{11}^{0}(\omega)=\frac{\omega}{\pi k} E\left(k^{2}\right)-\frac{\left(2+0.5 \omega^{2}\right) k \omega}{2 \pi} K\left(k^{2}\right)+\frac{1}{2}, \\
& F_{2}(x)= \begin{cases}\frac{4 B_{0}^{2} r^{2} \tau}{\mu_{0}}\left[\frac{1}{l_{1}}\left\{E\left(l_{1}^{2}\right)-K\left(l_{1}^{2}\right)\right\}-\frac{1}{l_{2}}\left\{E\left(l_{2}^{2}\right)-K\left(l_{2}^{2}\right)\right\}\right], & x=0, \\
\frac{\pi r^{2} B_{0}^{2}}{2 \mu_{0}}\left[A_{11}^{0}(z-2 \tau)-A_{11}^{0}(z)+A_{11}^{0}(z+2 \tau)\right], & x>0,\end{cases}
\end{aligned}
$$

where $K$ and $E$ are complete elliptic integrals of the first and the second kind respectively. By using the remnant magnetic field $\left(B_{0}\right)$ as a free parameter, the $F_{2}$ can be fitted with the experimental result with $B_{0}=1.357 \mathrm{~T}$, and the contact force (in the case $x=0$ ) is predicted as $351.5 \mathrm{~N}$. However, the $B_{0}$ is higher than the remnant magnetic field of common neodymium iron boron magnets.

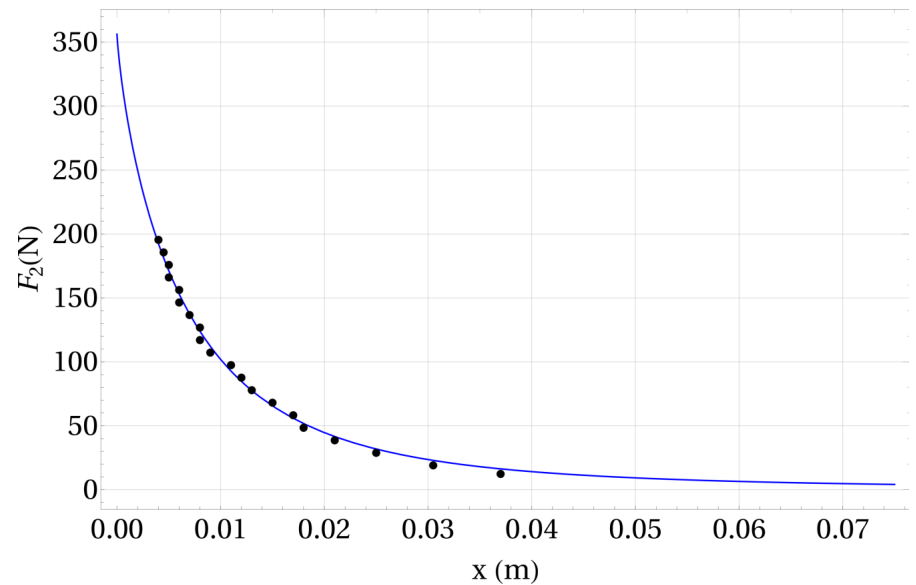

Fig. 2. Graph of fitting data points from the magnetic force measurement with $F_{2}$ from (2)

In [3], Camacho and Sosa suggested an alternative method to predict the magnetic force between two cylindrical magnet bars. However, the closed-form solution of magnetic force has not been proposed in [3]. By following the method in [3], the equation describing repulsive force $F_{3}$ between two identical cylindrical magnets as a function of their separation can be written as:

$$
F_{3}(x)=\frac{\pi r^{2} B_{0}^{2}}{2 \mu_{0}}\left[\frac{2(x+t)}{\sqrt{(x+t)^{2}+r^{2}}}-\frac{x+2 t}{\sqrt{(x+2 t)^{2}+r^{2}}}-\frac{x}{\sqrt{x^{2}+r^{2}}}\right]
$$

where all variables are defined as the same as in (2).

The (3) is derived from the magnetic field on the $z$-axis of the lower magnet which according to [3], can be obtained as:

$$
B(z)=\frac{\mu_{0} M}{2}\left[\frac{z}{\sqrt{z^{2}+r^{2}}}-\frac{z-t}{\sqrt{(z-t)^{2}+r^{2}}}\right] .
$$

The repulsive force between magnets has been defined as the volume integration on the upper magnet which can be obtained as:

$$
F_{3}=\int M_{2} \frac{\partial B(z)}{\partial z} \mathrm{~d} V
$$




$$
\begin{gathered}
d F_{3}=M_{2} \frac{\partial B(z)}{\partial z} d V=-M \frac{\partial B(z)}{\partial z} \pi r^{2} d z \\
\beta(z)=\frac{z}{\sqrt{z^{2}+r^{2}}}-\frac{z-t}{\sqrt{(z-t)^{2}+r^{2}}}, d F_{3}=-\frac{\pi r^{2} B_{0}^{2}}{2 \mu_{0}} d \beta(z) .
\end{gathered}
$$

From (7), the $F_{3}$ can be obtained as:

$$
\begin{gathered}
F_{3}=-\frac{\pi r^{2} B_{0}^{2}}{2 \mu_{0}} \int_{z=x+t}^{z=x+2 t} \mathrm{~d} \beta(z), \\
F_{3}=-\frac{\pi r^{2} B_{0}^{2}}{2 \mu_{0}}\left[\frac{z}{\sqrt{z^{2}+r^{2}}}-\frac{z-t}{\sqrt{(z-t)^{2}+r^{2}}}\right]_{z=x+t}^{z=x+2 t} .
\end{gathered}
$$

After the substitution of the upper and lower limits in (9), the expression as shown in (3) is obtained. Fig. 3 shows a same variation of the repulsive forces measured from the experiment and predicted by (3). In this graph fitting, the $B_{0}=1.206 \mathrm{~T}$, and the contact force is equal to $277.2 \mathrm{~N}$. The practical $B_{0}$ value of less than $1.3 \mathrm{~T}$ indicates the validity of the model.

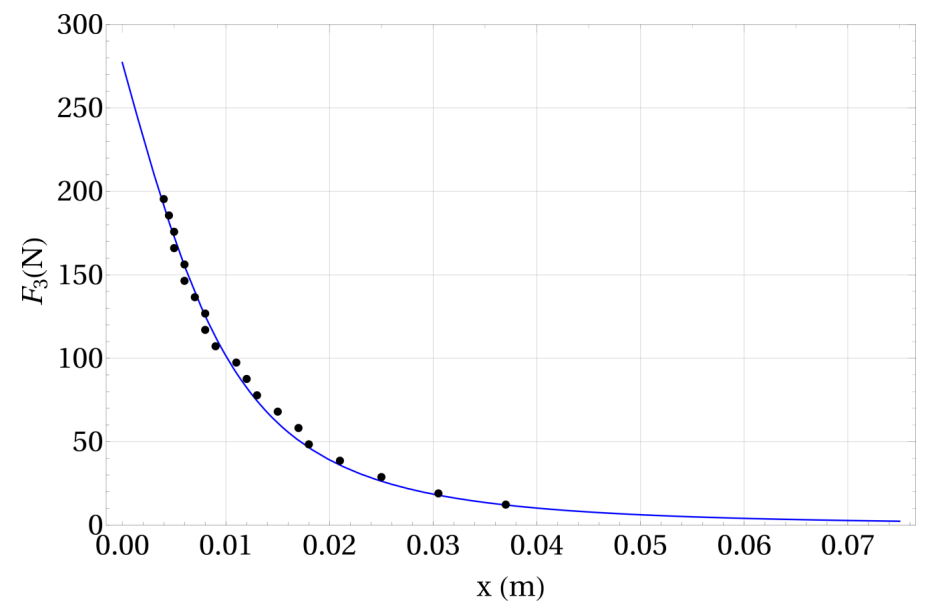

Fig. 3. Graph of fitting data points from the magnetic force measurement with $F_{3}$ from (3)

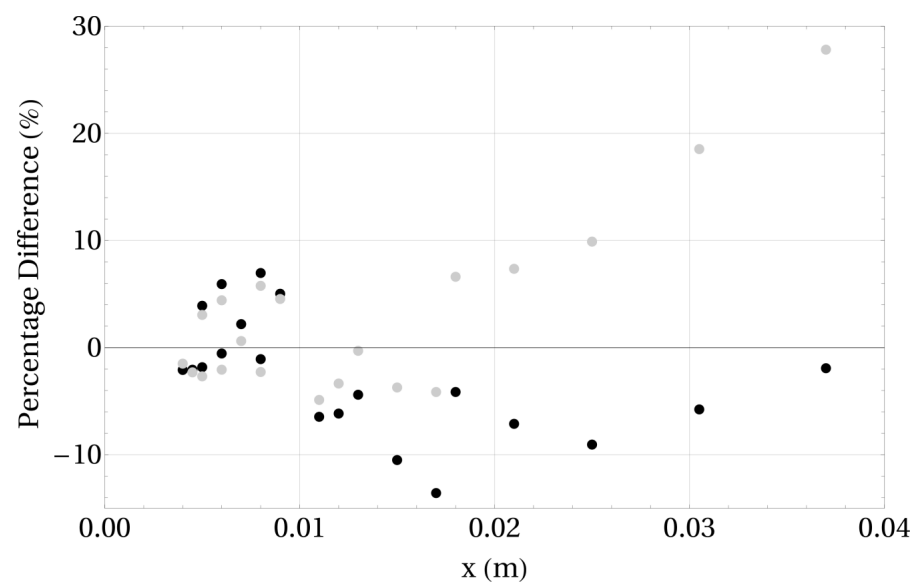

Fig. 4. Percentage of difference of the calculated forces, $F_{2}$ using (2) in grey dots and $F_{3}$ using (3) in black dot, from the experimental values measured from $x=0.004-0.037 \mathrm{~m}$ 
The percentage of difference between the experimental forces and values from equations are shown in Fig. 4. When $x$ is less than $0.009 \mathrm{~m}$, the values from both equations are comparable and differ from the experimental values by only $-0.55 \%$ to $-2.68 \%$. For (2), the difference is $6.61 \%$ in the case of $x=0.018 \mathrm{~m}$ and substantially rises with further increase in $x$. By contrast, the values obtained from (3) are lower than the experimental results with $x=0.011-0.037 \mathrm{~m}$ and the difference ranges from $-1.92 \%$ to $-13.60 \%$.

\section{Discussion of experimental results}

The agreement between the closed-form solution in (3) and the experimental measurement shown in Fig. 3 and their percentage difference in Fig. 4 suggest that the magnetic force between two cylindrical magnets can be calculated by an analytical treatment assuming uniform magnetizations along their length. For a small range of distance from 0.004 to $0.037 \mathrm{~m}$, a large magnitude of force was verified in this experiment.

The force in (3) can then be implemented with a better accuracy than a simple form in (1) to design structures and systems with small distances between permanent magnets. In the case of large distances, (3) is reduced to (1). When $t>r$ and $x \gg t$,

$$
\begin{aligned}
& F_{3}(x) \approx \frac{\pi r^{4} B_{0}^{2}}{4 \mu_{0} x^{2}}\left[\left\{2-4\left(\frac{t}{x}\right)+6\left(\frac{t}{x}\right)^{2}\right\}-\left\{1-2\left(\frac{2 t}{x}\right)+3\left(\frac{2 t}{x}\right)^{2}\right\}-1\right], \\
& F_{3}(x) \approx-\frac{3 \pi r^{4} t^{2} B_{0}^{2}}{2 \mu_{0} x^{4}}=-\frac{3 \mu_{0}\left(\frac{B_{0}}{\mu_{0}}\right)^{2}\left(\pi r^{2} t\right)^{2}}{2 \pi x^{4}}=-\frac{3 \mu_{0}(M V)^{2}}{2 \pi x^{4}}, \\
& F_{3} \approx \frac{3 \mu_{0} m_{1} m_{2}}{2 \pi x^{4}},
\end{aligned}
$$

where $m_{1}=-m_{2}=M V, V$ is volume of the upper magnet. In this regime, the expression in (3) exhibits a smaller variation from the experimental force than those obtained from (2).

The limitation of this study is that only one geometry of one type of permanent magnet was experimentally tested. Either change of composition or type (e. g. ferrite, samarium cobalt) of the magnets should affect the magnitude of the force measured and remnant field in the fitting but unlikely modify the shape of the curve. The different shapes of magnets were previously compared in [8]. It is demonstrated that the magnetic dipole-dipole model is accurate in the case of spherical magnets. However, the force between pairs of cuboid or cylindrical magnets are better described by magnetic charge and magnetizing current models.

The experimental results can directly be implemented in the levitation by permanent magnets. The weights up to $20 \mathrm{~kg}$ were lifted in the using small cylindrical neodymium iron boron magnets. Further calculations of magnetic field associated with such strong forces can be used to design new electrical generators, motors, flywheels, and magnetic refrigerators.

\section{Conclusions}

The force between two cylindrical neodymium iron boron magnets is measured as a function of distance and described by a closed-form solution. In the $0.004-0.037 \mathrm{~m}$ regime, this presented analytic solution and the experimental magnetic force exhibit the same trend with the percentage difference ranging from $-0.55 \%$ to $-13.60 \%$. The contact force can also be estimated. By using the remnant magnetic field $\left(B_{0}\right)$ as a free parameter, the $B_{0}=1.206 \mathrm{~T}$ from the fitting is comparable to a practical value supplied by neodymium iron boron type magnets. If the separation distance $x$ between magnets is far enough, the force between magnets in this equation is approximately proportional to $x^{-4}$. The equations and findings are useful for optimizing the configuration of magnets in engineering devices. 


\section{References}

[1] Martins, R. de A. (2017). O estudo experimental sobre o magnetismo na Idade Média, com uma tradução da carta sobre o magneto de Petrus Peregrinus. Revista Brasileira de Ensino de Física, 39 (1), e1601. doi: http://doi.org/10.1590/1806-9126rbef-2016-0181

[2] Vokoun, D., Beleggia, M., Heller, L., Šittner, P. (2009). Magnetostatic interactions and forces between cylindrical permanent magnets. Journal of Magnetism and Magnetic Materials, 321 (22), 3758-3763. doi: http://doi.org/10.1016/j.jmmm.2009.07.030

[3] Camacho, M., Sosa, V. (2013). Alternative method to calculate the magnetic field of permanent magnets with azimuthal symmetry. Revista Mexicana de Fisica E 59, 8-17.

[4] Robertson, W., Cazzolato, B., Zander, A. (2011). A simplified force equation for coaxial cylindrical magnets and thin coils. IEEE Transactions on Magnetics, 47 (8), 2045-2049. doi: http://doi.org/10.1109/tmag.2011.2129524

[5] Ravaud, R., Lemarquand, G., Babic, S., Lemarquand, V., Akyel, C. (2010). Cylindrical magnets and coils: Fields, forces, and inductances. IEEE Transactions on Magnetics, 46 (9), 3585-3590. doi: http://doi.org/10.1109/tmag.2010.2049026

[6] Allag, H., Yonnet, J.-P. (2009). 3-D analytical calculation of the torque and force exerted between two cuboidal magnets. IEEE Transactions on Magnetics, 45 (10), 3969-3972. doi: http://doi.org/10.1109/tmag.2009.2025047

[7] Agashe, J. S., Arnold, D. P. (2008). A study of scaling and geometry effects on the forces between cuboidal and cylindrical magnets using analytical force solutions. Journal of Physics D: Applied Physics, 41 (10), 105001. doi: http://doi.org/10.1088/ 0022-3727/41/10/105001

[8] Zhang, Y., Leng, Y., Zhang, H., Su, X., Sun, S., Chen, X., Xu, J. (2020). Comparative study on equivalent models calculating magnetic force between permanent magnets. Journal of Intelligent Manufacturing and Special Equipment, 1 (1), $43-65$. doi: http://doi.org/10.1108/jimse-09-2020-0009

[9] Liu, Z. J., Li, J. T. (2008). Accurate prediction of magnetic field and magnetic forces in permanent magnet motors using an analytical solution. IEEE Transactions on Energy Conversion, 23 (3), 717-726. doi: http://doi.org/10.1109/tec.2008.926034

[10] Vokoun, D., Tomassetti, G., Beleggia, M., Stachiv, I. (2011). Magnetic forces between arrays of cylindrical permanent magnets. Journal of Magnetism and Magnetic Materials, 323 (1), 55-60. doi: http://doi.org/10.1016/j.jmmm.2010.08.029

[11] Schomburg, W. K., Reinertz, O., Sackmann, J., Schmitz, K. (2020). Equations for the approximate calculation of forces between cuboid magnets. Journal of Magnetism and Magnetic Materials, 506, 166694. doi: http://doi.org/10.1016/j.jmmm.2020.166694

[12] Samoh, A., Niamjan, N., Yaiprasert, C., Sirisathitkul, Y., Sirisathitkul, C. (2019). Comsol simulations of magnetic flux generated by permanent magnets with ring geometries. Journal of Science and Arts, 19, 775-782.

[13] Shvedchykova, I., Melkonova, I., Romanchenko, J. (2020). Research of magnetic field distribution in the working area of disk separator, taking into account an influence of materials of permanent magnets. EUREKA: Physics and Engineering, 1, 87-95. doi: http://doi.org/10.21303/2461-4262.2020.001106

[14] Nonlinear Least Squares Fitting. Available at: http://mathworld.wolfram.com/NonlinearLeastSquaresFitting.html

Received date 13.04.2021

Accepted date 14.07.2021

Published date 23.07.2021
(C) The Author(s) 2021

This is an open access article under the Creative Commons CC BY license

How to cite: Cheedket, S., Sirisathitkul, C. (2021). Comparison of closed-form solutions to experimental magnetic force between two cylindrical magnets. EUREKA: Physics and Engineering, 4, 141-146. http://doi.org/10.21303/2461-4262.2021.001955 